\title{
Renal Lymphoma. Atypical Presentation of a Renal Tumor
}

\author{
Francualdo Barreto, Marcos F. Dall'Oglio, Miguel Srougi \\ Department of Urology, Federal University of Sao Paulo (UNIFESP), Paulista School of Medicine, \\ Sao Paulo, Brazil
}

\begin{abstract}
Primary renal lymphoma is a rare lesion that represents less than $1 \%$ of the kidney's lesions. The authors discuss the case of a 67-year-old woman with a renal mass identified 7 years after treatment of a non-Hodgkin's lymphoma, and analyze clinical and prognostic aspects of renal lymphomas. Radiological findings in this case showed an uncommon presentation of the renal lymphomatous lesion which served as a warning that tumors might appear during follow-up as atypical and uncommon lesions.
\end{abstract}

Key words: kidney neoplasms; nephrectomy; lymphoma

Int Braz J Urol. 2006; 32: 190-2

\section{INTRODUCTION}

A primary renal lymphoma is a rare lesion that represents less than $1 \%$ of the lesions in this organ (1). Renal lymphoma has an insidious clinical presentation that occurs late in the course of the disease $(2,3)$. It can present in many ways, however the most common are primary tumors presenting single or multiple nodules, or that involve the kidney, either in a hematogenic dissemination form or through a contiguous retroperineal disease (2).

The authors discuss the case of a 67 -year-old patient presenting a solitary renal mass 7 years after the chemotherapy and radiotherapy treatment of a non-Hodgkin's lymphoma.

\section{CASE REPORT}

A 67-year-old female asymptomatic patient with no palpable lymph nodes presented a right renal mass (Figure-1) in an abdomen computerized tomography $(\mathrm{CT})$ in a routine check up for a non-Hodgkin's lymphoma that had been diagnosed and treated with chemotherapy and radiotherapy 7 years earlier.

The patient underwent a radical right nephrectomy and the macroscopic exam revealed an expansion of fat caused by a homogeneous, yellowish tumor infiltration. The microscopic exam revealed a centrofollicular lymphoma, follicular grade I, diffusely infiltrating the renal interstitium. The immunohistochemical analysis demonstrated a CD 20 positivity, characterizing a lymphoma originating in lymphocytes B (Figure-2). This finding led to adjuvant radiotherapy and the patient is now with a 16-month follow-up.

\section{COMMENTS}

Among cases of renal lymphoma, between $37 \%$ and $47 \%$ occur due to dissemination of an ad- 


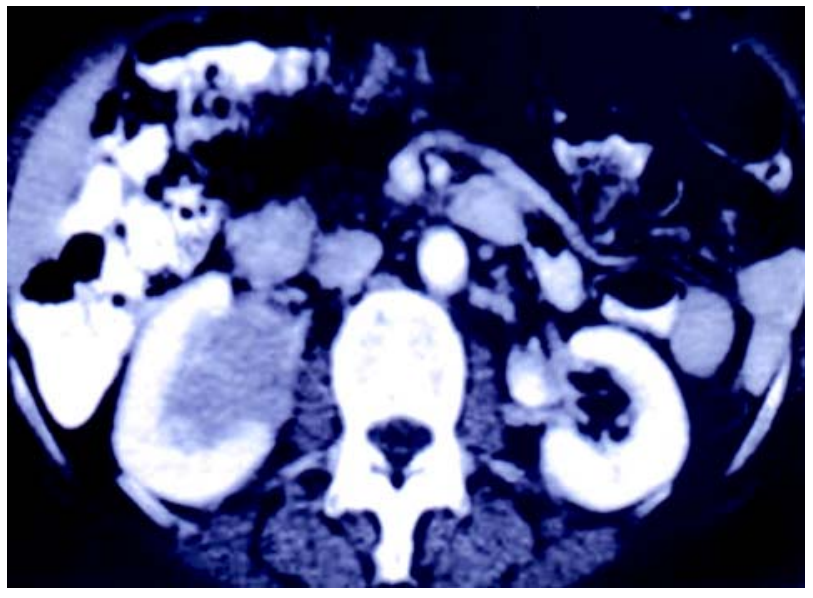

Figure 1 - A four-centimeter solid and intra-sinusal right renal mass with contrast enhancement.

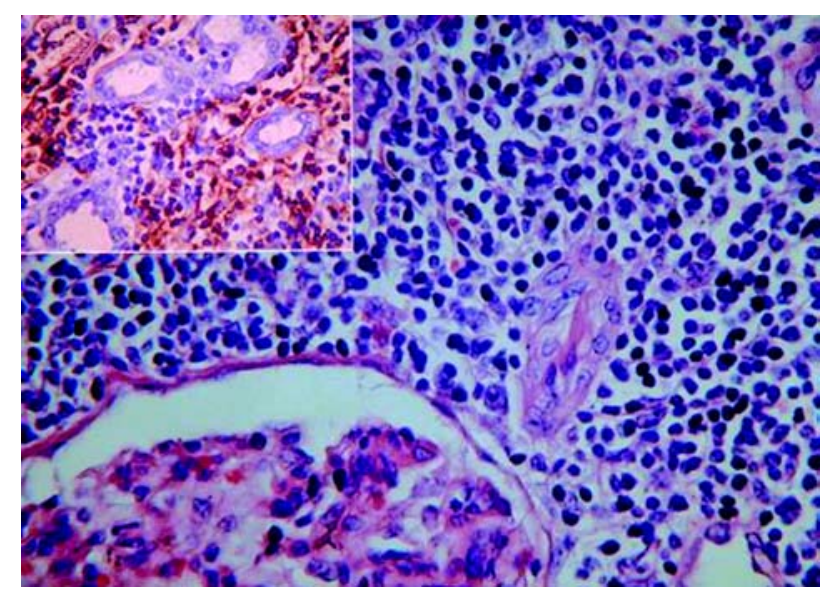

Figure 2 - Centrofollicular lymphoma, follicular grade I diffusely infiltrating the renal interstitium (HE). Insert: Immunohistochemical study demonstrating the CD2O expression, characterizing a cell $B$ origing lymphoma.

vanced systemic disease (2), while $0.1 \%$ are due to primary involvement of the kidney (1). The term, primary renal lymphoma, can be used either when the initial manifestation involves the kidney or the lesion is limited to it (3).

Renal lymphoma usually is of small cells (Burkitt's), or less frequently of lymphocytes B (1). Prognosis of these cases is not well established, but is typically defined using the criteria of the Working Formulation, Ann Arbor Stage and International Prognostic Index (3). The disease has the same prevalence in both sexes and is predominant in patients with a mean age of 66 years (3). The symptoms observed during the later stages are lumbalgia, hematuria and fever (3). However, in the series studied by Dimopoulos et al., all 6 patients presented symptoms related to the involvement of the urinary tract and the absence of peripheral palpable lymph nodes (3). Even with chemotherapy, only 2 patients had complete remission of the disease. The patients with unfavorable prognostic factors as defined by the International Prognostic Index presented poor results with chemotherapy treatment (3), which is based on cyclofosfamide, doxorubicin, vincristine and prednisone.

Lesions can be solitary masses (10-20\%) or multiple masses $(60 \%)$. They are generally bilateral and present extension by contiguity (25\%-30\%), diffuse infiltration (20\%) or perirenal involvement $10 \%$ (2). Radiological findings frequently indicate renal involvement with multiple nodules (60\%) and help in clarifying the diagnosis when considered along with previous family history. Renal lymphoma generally is presented as a bilateral nodular infiltration with a diffuse kidney increase and infiltration of the renal parenchyma by a diffuse invasion of the retroperitoneum (3). Solitary unilateral renal mass, perirenal mass with distortion of the renal architecture in the CT scan and absence of lymph node enlargements are more suggestive of renal cell carcinomas (3), however the presence of solid masses can require a biopsy to rule out other pathologies (2).

Radiological findings in this case showed an uncommon presentation of the renal lymphomatous lesion which served as a warning that tumors might appear during follow-up as atypical and uncommon lesions.

\section{CONFLICT OF INTEREST}

None declared.

\section{REFERENCES}

1. Stallone G, Infante B, Manno C, Campobasso N, Pannarale G, Schena FP: Primary renal lymphoma does 


\section{Renal Lymphoma}

exist: case report and review of the literature. J Nephrol. 2000; 13: 367-72.

2. Urban BA, Fishman EK: Renal lymphoma: CT patterns with emphasis on helical CT. Radiographics. 2000; 20: 197-212.
3. Dimopoulos MA, Moulopoulos LA, Costantinides C, Deliveliotis C, Pantazopoulos D, Dimopoulos C: Primary renal lymphoma: a clinical and radiological study. J Urol. 1996; 155: 1865-7.

Accepted after revision: August 31, 2005

Correspondence address:

Dr. Marcos F Dall'Oglio

Rua Manoel da Nóbrega, 853 / 22

São Paulo, SP, 04001-084, Brazil

Fax: + $55113885-0658$

E-mail: marcosdallogliouro@terra.com.br 